\title{
Exact solution and dynamic buckling analysis of a beam-column system having the elliptic type loading*
}

\author{
H. S. ARTEM, L. AYDIN \\ (Department of Mechanical Engineering, Izmir Institute of Technology, \\ Gulbahce Koyu, Urla-Izmir 35430, Turkey) \\ (Communicated by Li-qun CHEN)
}

\begin{abstract}
This paper presents a closed form solution to the dynamic stability problem of a beam-column system with hinged ends loaded by an axial periodically time-varying compressive force of an elliptic type, i.e., $a_{1} \mathrm{cn}^{2}\left(\tau, k^{2}\right)+a_{2} \operatorname{sn}^{2}\left(\tau, k^{2}\right)+a_{3} \mathrm{dn}^{2}\left(\tau, k^{2}\right)$. The solution to the governing equation is obtained in the form of Fourier sine series. The resulting ordinary differential equation is solved analytically. Finding the exact analytical solutions to the dynamic buckling problems is difficult. However, the availability of exact solutions can provide adequate understanding for the physical characteristics of the system. In this study, the frequency-response characteristics of the system, the effects of the static load, the driving forces, and the frequency ratio on the critical buckling load are also investigated.
\end{abstract}

Key words dynamic buckling, exact solution, stability-instability, Jacobi elliptic functions

Chinese Library Classification O311.3

2000 Mathematics Subject Classification 74D05, 74K10, 33E05, 34B30, 70E50

\section{Introduction}

The analysis of the dynamic behavior of beam-column systems is of great importance in structural and engineering design. If a structural component is subjected to a dynamic loading, the dynamic buckling problem can be observed. The dynamic stability depends on the applied force and the geometry of the structure. In this study, the exact solution of the beam-column system subjected to a pulsating load of an elliptic type is considered. The pulsating load can often be approximate to the action of rotating machinery, i.e., turbines and power generators, on the columns of structures ${ }^{[1]}$.

A few researchers have studied the analytical solution to dynamic stability problems. The stability and the dynamic analysis of a two-dimensional shear beam-column with generalized boundary conditions have been studied by Aristizabal-Ochoa ${ }^{[2]}$. The analytical results showed that the stability and the dynamic behavior of shear beams and shear beam-columns were governed by the bending moment equation. Pavlovic et al. ${ }^{[3]}$ considered the dynamic stability problem of thin-walled beams subjected to the combined action of axial loads and end moments.

* Received Nov. 2, 2009 / Revised Jul. 8, 2010

Corresponding author H. S. ARTEM, Ph. D., E-mail: secilartem@iyte.edu.tr 
Closed form analytical solutions were obtained for simply supported boundary conditions. By using the direct Lyapunov method, almost sure asymptotic stability and uniform stochastic stability conditions were obtained as a function of stochastic process variance, damping coefficient, geometric, and physical parameters of the beam. The inclined beam-column theory, incorporating distributed weight, and the inclination of the beam-column system have been investigated by Sampaio and Hundhausen ${ }^{[4]}$. They used an energy method to obtain the differential equation of the system, and a closed form solution was obtained in terms of hypergeometric functions. Zuniga $^{[5]}$ studied the problem of the dynamic response of a beam-column system with hinged ends subjected to an axial pulsating force of an elliptic type, i.e., $a \mathrm{cn}^{2}\left(\tau, k^{2}\right)$. The general exact solution to the resulting equation, that is, the well-known Lamé equation, was obtained for the hinged-hinged beam-column system.

The objectives of the present study are as follows:

(i) to find the analytical solution to the Lamé equation,

$$
\frac{d^{2} u_{1}}{d \tau^{2}}+u_{1}(\tau)\left(d_{1}+d_{2 \mathrm{c}} \mathrm{cn}^{2}\left(\tau, k^{2}\right)+d_{2 \mathrm{~s}} \mathrm{sn}^{2}\left(\tau, k^{2}\right)+d_{2 \mathrm{~d}} \mathrm{dn}^{2}\left(\tau, k^{2}\right)\right)=0
$$

(ii) to investigate the dynamic response characteristics of a beam-column system with hinged ends subjected to the time-varying force of an elliptic type,

$$
a_{1} \mathrm{cn}^{2}\left(\tau, k^{2}\right)+a_{2} \operatorname{sn}^{2}\left(\tau, k^{2}\right)+a_{3} \mathrm{dn}^{2}\left(\tau, k^{2}\right) .
$$

\section{Elastic beam-column with hinged ends loaded by an axial time-varying force}

A dynamic stability of a uniform beam-column system with hinged ends is considered as shown in Fig. 1.

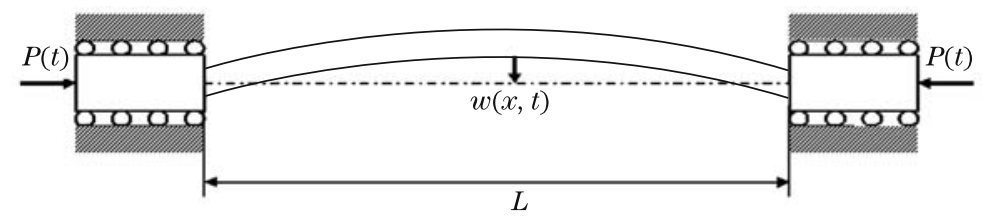

Fig. 1 Beam-column system subjected to a time-varying force

Both ends of this system are subjected to an axial time-varying compressive force of

$$
P(t)=P_{0}+P_{1}(t)
$$

where $P_{0}$ is a stationary force, and $P_{1}(t)$ is a time-varying force of an elliptic type. The governing equation of the beam-column system is

$$
\rho A \frac{\partial^{2} w}{\partial t^{2}}+P(t) \frac{\partial^{2} w}{\partial x^{2}}+E I \frac{\partial^{4} w}{\partial x^{4}}=0
$$

Equation (2) should be solved together with the following boundary conditions:

$$
\begin{aligned}
& w(0, t)=w(L, t)=0, \\
& \frac{\partial^{2}}{\partial x^{2}} w(0, t)=\frac{\partial^{2}}{\partial x^{2}} w(L, t)=0,
\end{aligned}
$$


where $w(x, t)$ defines the lateral deflection. $\rho$ is the density per unit length. $E, I$, and $A$ represent the modulus of elasticity, the moment of inertia, and the cross sectional area, respectively. The solution to the problem can be obtained conveniently by taking $w(x, t)$ as Fourier sine series

$$
w(x, t)=\sum_{n=1}^{\infty} u_{n}(t) \sin \left(\frac{n \pi x}{L}\right) .
$$

Substitute Eq. (5) into Eq. (2). $u_{n}(t)$ can be obtained by the following system of the linear differential equation:

$$
\sum_{n=1}^{\infty}\left(\rho A \ddot{u}_{n}(t)-P(t)\left(\frac{n \pi}{L}\right)^{2} u_{n}(t)+E I\left(\frac{n \pi}{L}\right)^{4} u_{n}(t)\right) \sin \left(\frac{n \pi x}{L}\right)=0 .
$$

Since only the lowest eigenvalue has physical significance in buckling problems, Eq. (6) should be rewritten for the fundamental mode $(n=1)$ as the following form:

$$
\left(\rho A \ddot{u}_{1}(t)-P(t)\left(\frac{\pi}{L}\right)^{2} u_{1}(t)+E I\left(\frac{\pi}{L}\right)^{4} u_{1}(t)\right) \sin \left(\frac{\pi x}{L}\right)=0 .
$$

To have a non-trivial solution to Eq. (7), the terms in the parentheses must be equal to zero, i.e.,

$$
\rho A \ddot{u}_{1}(t)-P(t)\left(\frac{\pi}{L}\right)^{2} u_{1}(t)+E I\left(\frac{\pi}{L}\right)^{4} u_{1}(t)=0 .
$$

Therefore, the problem is reduced to a second-order ordinary differential equation. Equation (8) may be non-dimensionalized for convenience in the numerical scheme by introducing dimensionless variable $\tau=\Omega t$ and defining the Euler load $P_{\mathrm{c}}=\pi^{2} E I / L^{2}$ and the frequency $\omega_{0}^{2}=\pi^{4} E I /\left(\rho A L^{4}\right)$. Then, the following differential equation with variable coefficients can be obtained:

$$
\frac{d^{2} u_{1}}{d \tau^{2}}+u_{1}(\tau)\left(d_{1}+\bar{d}_{1} P_{1}(\tau)\right)=0
$$

where

$$
d_{1}=\frac{\omega_{0}^{2}}{\Omega^{2}}\left(1-\frac{P_{0}}{P_{\mathrm{c}}}\right), \quad \bar{d}_{1}=\frac{-\omega_{0}^{2}}{\Omega^{2} P_{\mathrm{c}}} .
$$

\section{Solution}

The periodically varying force of $P_{1}(\tau)$ appearing in Eq. (9) is assumed as the linear combination of squares of Jacobi elliptic functions $\operatorname{cn}\left(\tau, k^{2}\right), \operatorname{sn}\left(\tau, k^{2}\right)$, and $\operatorname{dn}\left(\tau, k^{2}\right)$ in the form of (the elementary definition of the elliptic functions is given in [6])

$$
P_{1}(\tau)=a_{1} \operatorname{cn}^{2}\left(\tau, k^{2}\right)+a_{2} \operatorname{sn}^{2}\left(\tau, k^{2}\right)+a_{3} \operatorname{dn}^{2}\left(\tau, k^{2}\right),
$$

where $a_{1}, a_{2}$, and $a_{3}$ are arbitrary real constants defining the magnitude of driving forces, and $k$ is the modulus. Note that $k$ should take the value between 0 and 1 , otherwise the elliptic functions will become complex. Therefore, the loading described by Eq. (11) will be physically meaningless. The behavior of $P(\tau)$ is shown in Fig. 2 for different values of loading parameters $a_{1}, a_{2}$, and $a_{3}$. When $a_{1}=1$ and $a_{2}=a_{3}=0, P(\tau)$ agrees with the force studied in [5].

Now, substituting Eq. (11) into Eq. (9), one obtains

$$
\frac{d^{2} u_{1}}{d \tau^{2}}+u_{1}(\tau)\left(d_{1}+d_{2 \mathrm{c}} \operatorname{cn}^{2}\left(\tau, k^{2}\right)+d_{2 \mathrm{~s}} \operatorname{sn}^{2}\left(\tau, k^{2}\right)+d_{2 \mathrm{~d}} \mathrm{dn}^{2}\left(\tau, k^{2}\right)\right)=0
$$

where

$$
d_{2 \mathrm{c}}=\bar{d}_{1} a_{1}, \quad d_{2 \mathrm{~s}}=\bar{d}_{1} a_{2}, \quad d_{2 \mathrm{~d}}=\bar{d}_{1} a_{3}
$$




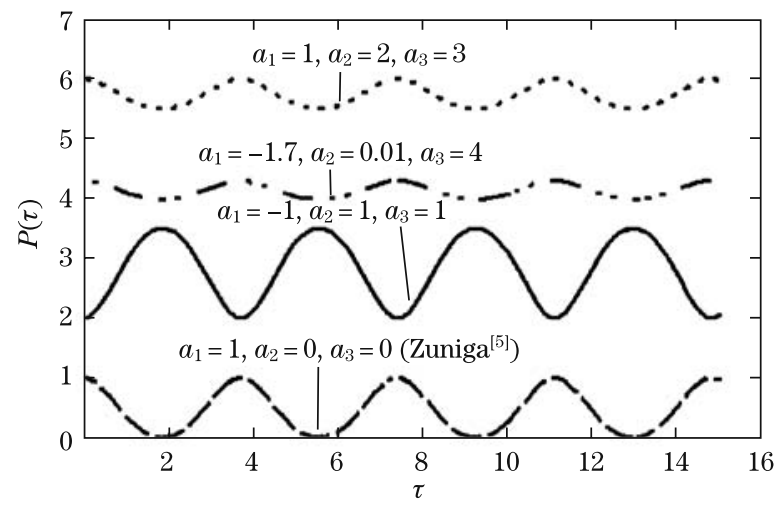

Fig. 2 Behavior of $P(\tau)$ for different values of $a_{1}, a_{2}$, and $a_{3}$ with a static load $P_{0}=2$ and modulus $k=\sqrt{2} / 2$

If $d_{2 \mathrm{~s}}=d_{2 \mathrm{~d}}=0$, Eq. (13) reduces to the Lamé equation studied in [5].

To find the general solution to Eq. (12), the formulation given in [7] can be used. In this formulation, a second-order linear ordinary differential equation should be in the form of

$$
f_{2}(x) \frac{d^{2} u}{d x^{2}}+f_{1}(x) \frac{d u}{d x}+f_{0}(x) u(x)=0
$$

and the general solution can be found as

$$
u(x)=u_{\mathrm{p}}\left(c_{1}+c_{2} \int \frac{\mathrm{e}^{-F}}{u_{\mathrm{p}}^{2}} d x\right),
$$

where

$$
F=\int \frac{f_{1}(x)}{f_{2}(x)} d x
$$

If a non-trivial particular solution $u_{\mathrm{p}}$ is known ${ }^{[7]}$, a particular solution can be found according to Eq. (15), which is a general solution to Eq. (12). To find a particular solution, the following criteria should be satisfied.

(i) It should be a function of Jacobi elliptic functions $\mathrm{cn}$, sn, and dn, since the derivatives of these three functions are always expressed in terms of the same elliptic functions.

(ii) The integrand given in Eq. (15) should be taken analytically in order to express the general solution expilicitly.

Considering all the criteria mentioned above, by trial and error, the following form can be introduced as a particular solution to Eq. (12):

$$
u_{1 \mathrm{p}}(\tau)=\sqrt{\operatorname{cn}\left(\tau, k^{2}\right)+\operatorname{dn}\left(\tau, k^{2}\right)} .
$$

Note that although the particular solution given in [5] also satisfies these criteria ((i) and (ii)), the resulting algebraic equation for Eq. (12) cannot be solved.

Substituting Eq. (17) into Eq. (12) and using the following relations:

$$
\operatorname{sn}\left(\tau, k^{2}\right)=\sqrt{1-\mathrm{cn}^{2}\left(\tau, k^{2}\right)}, \quad \operatorname{dn}\left(\tau, k^{2}\right)=\sqrt{1-k^{2}+k^{2} \mathrm{cn}^{2}\left(\tau, k^{2}\right)},
$$

one obtains Eq. (12) as the function of $\mathrm{cn}^{2}\left(\tau, k^{2}\right)$ in the following form:

$$
\begin{aligned}
& \left(-1+4 d_{1}+4 d_{2 \mathrm{~s}}+4 d_{2 \mathrm{~d}}+2 k^{2}-4 d_{2 \mathrm{~d}} k^{2}+4 d_{2 \mathrm{c}} \mathrm{cn}^{2}\left(\tau, k^{2}\right)-4 d_{2 \mathrm{~s}} \mathrm{cn}^{2}\left(\tau, k^{2}\right)\right. \\
& \left.-3 k^{2} \mathrm{cn}^{2}\left(\tau, k^{2}\right)+4 d_{2 \mathrm{~d}} k^{2} \mathrm{cn}^{2}\left(\tau, k^{2}\right)\right)\left(1-k^{2}+\mathrm{cn}^{2}\left(\tau, k^{2}\right)+k^{2} \operatorname{cn}^{2}\left(\tau, k^{2}\right)\right. \\
& \left.+2 \operatorname{cn}\left(\tau, k^{2}\right) \sqrt{1-k^{2}+k^{2} \operatorname{cn}^{2}\left(\tau, k^{2}\right)}\right) /\left(4 \operatorname{cn}\left(\tau, k^{2}\right)+4 \sqrt{1-k^{2}+k^{2} \operatorname{cn}^{2}\left(\tau, k^{2}\right)}\right)^{\frac{3}{2}}=0 .
\end{aligned}
$$


The above algebraic equation is to be valid for all values of $\tau$ if the following conditions are satisfied:

$$
\begin{aligned}
& -1+4 d_{1}+4 d_{2 \mathrm{~s}}+4 d_{2 \mathrm{~d}}+2 k^{2}-4 d_{2 \mathrm{~d}} k^{2}=0, \\
& 4 d_{2 \mathrm{c}}-4 d_{2 \mathrm{~s}}-3 k^{2}+4 d_{2 \mathrm{~d}} k^{2}=0 .
\end{aligned}
$$

Then, the general solution satisfying the conditions given by Eqs. (20) and (21) can now be obtained in the form of

$$
u_{1}(\tau)=u_{1 \mathrm{p}}\left(c_{1}+c_{2} \int \frac{\mathrm{e}^{-m}}{u_{1 \mathrm{p}}^{2}(\tau)} d \tau\right)
$$

where $m$ is an arbitrary constant.

The integral appearing in Eq. $(22)$ is obtained ${ }^{[8]}$. Therefore, the general solution is

$$
u_{1}(\tau)=\sqrt{\operatorname{cn}\left(\tau, k^{2}\right)+\operatorname{dn}\left(\tau, k^{2}\right)}\left(c_{1}+c_{0} \frac{\operatorname{sn}\left(\tau, k^{2}\right)}{\operatorname{cn}\left(\tau, k^{2}\right)+\operatorname{dn}\left(\tau, k^{2}\right)}\right),
$$

where $c_{0}=\mathrm{e}^{-m} c_{2}$.

From the initial conditions

$$
u_{1}(0)=u_{1 \mathrm{c}}, \quad \frac{d u_{1}}{d \tau}(0)=\bar{u}_{1 \mathrm{c}}
$$

where $u_{1 \mathrm{c}}$ and $\bar{u}_{1 \mathrm{c}}$ are arbitrary constants, we find the exact solution

$$
u_{1}(\tau)=\sqrt{\operatorname{cn}\left(\tau, k^{2}\right)+\operatorname{dn}\left(\tau, k^{2}\right)}\left(\frac{u_{1 \mathrm{c}}}{\sqrt{2}}+\bar{u}_{1 \mathrm{c}} \sqrt{2} \frac{\operatorname{sn}\left(\tau, k^{2}\right)}{\operatorname{cn}\left(\tau, k^{2}\right)+\operatorname{dn}\left(\tau, k^{2}\right)}\right) .
$$

Equation (25) includes bounded and unbounded terms. In order to make the solution physically meaningful, unbounded terms need to be vanished. For this purpose, appropriate initial conditions are introduced as $u_{1}(0)=u_{1 \mathrm{c}}$ and $d u_{1}(0) / d \tau=0$.

Therefore, the solution to the Lamé equation becomes bounded, and is in the form of

$$
u_{1 \mathrm{~b}}=\frac{u_{1 \mathrm{c}}}{\sqrt{2}} \sqrt{\operatorname{cn}\left(\tau, k^{2}\right)+\operatorname{dn}\left(\tau, k^{2}\right)} .
$$

In the second part of this study, the frequency-response characteristics of the system are discussed. For this purpose, using Eqs. (10), (13), (20), and (21), the relation related to the Euler load $P_{\mathrm{c}}$, driving forces $a_{1}, a_{2}$, and $a_{3}$, the static load $P_{0}$, and the frequency ratio $\omega_{0} / \Omega$ are obtained after expanding algebraic manipulations as

$$
\frac{3 P_{\mathrm{c}}^{2}+4\left(2 a_{1}+a_{2}+4 a_{3}\right) P_{\mathrm{c}}\left(\frac{\omega_{0}}{\Omega}\right)^{2}+16 a_{3}\left(a_{1}+a_{3}\right)\left(\frac{\omega_{0}}{\Omega}\right)^{4}}{4 P_{\mathrm{c}}\left(3 P_{\mathrm{c}}+4 a_{\mathrm{c}}\left(\frac{\omega_{0}}{\Omega}\right)^{2}\right)}=\left(\frac{\omega_{0}}{\Omega}\right)^{2}\left(1-\frac{P_{0}}{P_{\mathrm{c}}}\right) .
$$

The equation given above is rearranged to observe the effect of loading parameters on the change of frequency ratio as

$$
\begin{aligned}
& 3 P_{\mathrm{c}}^{2} /\left(4 a_{1} P_{\mathrm{c}}+2 a_{2} P_{\mathrm{c}}+8 a_{3} P_{\mathrm{c}}+6 P_{0} P_{\mathrm{c}}-3 P_{\mathrm{c}}^{2}\right. \\
& \left.+2 \sqrt{\left(\left(2 a_{1}+a_{2}+4 a_{3}+3 P_{0}-3 P_{\mathrm{c}}\right)^{2}-12 a_{3}\left(a_{1}+a_{3}+P_{0}-P_{\mathrm{c}}\right)\right) P_{\mathrm{c}}^{2}}\right) \\
& =\frac{\left(\frac{\omega_{0}}{\Omega}\right)^{2}}{\left(\frac{\omega_{0}}{\Omega}\right)^{2}-1} .
\end{aligned}
$$


The left-hand side of Eq. (28) will be represented by $F\left(P_{\mathrm{c}}, P_{0}, a_{1}, a_{2}, a_{3}\right)$ for better presentation in the related table and figures. Frequency response characteristics for different values of $a_{1}, a_{2}$, and $a_{3}$ are tabulated in Table 1 . The values in the first line of the table agree with the frequency reponse discussed in [5]. It is seen that the difference in the frequency response characteristics between the present study and that of Zuniga ${ }^{[5]}$ results from different analytical solutions.

Table 1 Special cases for frequency response characteristic $F\left(P_{\mathrm{c}}, P_{0}, a_{1}, a_{2}, a_{3}\right)$

\begin{tabular}{llll}
\hline$a_{1}$ & $a_{2}$ & $a_{3}$ & $F\left(P_{\mathrm{c}}, P_{0}, a_{1}, a_{2}, a_{3}\right)$ \\
\hline$a_{1}$ & 0 & 0 & $3 P_{\mathrm{c}}\left(8 a_{1}+12 P_{0}-9 P_{\mathrm{c}}\right)^{-1}\left(\mathrm{Zuniga}^{[5]}\right)$ \\
0 & $a_{2}$ & $a_{3}$ & $3 P_{\mathrm{c}}^{2}\left(2 a_{2} P_{\mathrm{c}}+8 a_{3} P_{\mathrm{c}}+6 P_{0} P_{\mathrm{c}}-3 P_{\mathrm{c}}^{2}\right.$ \\
& 0 & $\left.+2 \sqrt{\left[\left(a_{2}+4 a_{3}+3 P_{0}-3 P_{\mathrm{c}}\right)^{2}-12 a_{3}\left(a_{3}+P_{0}-P_{\mathrm{c}}\right)\right] P_{\mathrm{c}}^{2}}\right)^{-1}$ \\
$a_{1}$ & $a_{3}$ & $3 P_{\mathrm{c}}^{2}\left(4 a_{1} P_{\mathrm{c}}+8 a_{3} P_{\mathrm{c}}+6 P_{0} P_{\mathrm{c}}-3 P_{\mathrm{c}}^{2}\right.$ \\
$a_{1}$ & $a_{2}$ & $\left.+2 \sqrt{\left[\left(2 a_{1}+4 a_{3}+3 P_{0}-3 P_{\mathrm{c}}\right)^{2}-12 a_{3}\left(a_{1}+a_{3}+P_{0}-P_{\mathrm{c}}\right)\right] P_{\mathrm{c}}^{2}}\right)^{-1}$ \\
\hline
\end{tabular}

\section{Results and discussions}

The frequency response characteristics of the beam-column system with hinged ends loaded by an axial periodically time-varying compressive force of an elliptic type given by Eq. (28) are shown in Fig. 3. In the interval $(0,1)$ for the ratio $\omega_{0} / \Omega, F\left(P_{\mathrm{c}}, P_{0}, a_{1}, a_{2}, a_{3}\right)$ asymptotically increases and goes to infinity at $\omega_{0} / \Omega=1$, where the resonance occurs. With the increase of $\omega_{0} / \Omega, F\left(P_{\mathrm{c}}, P_{0}, a_{1}, a_{2}, a_{3}\right)$ approaches asymptotically to one.

The effects of driving forces $a_{1}, a_{2}$, and $a_{3}$ on $P_{c}$ with the values of the frequency ratio $\omega_{0} / \Omega=0.6$ and the static load $P_{0}=1$ are shown in Fig. $4 . P_{\mathrm{c}}$ shows linearly increasing behavior with respect to $a_{1}, a_{2}$, and $a_{3}$. It is observed from the figure that change in $P_{\mathrm{c}}$ with $a_{3}$ is more obvious compared with the other driving forces $a_{1}$ and $a_{2}$.

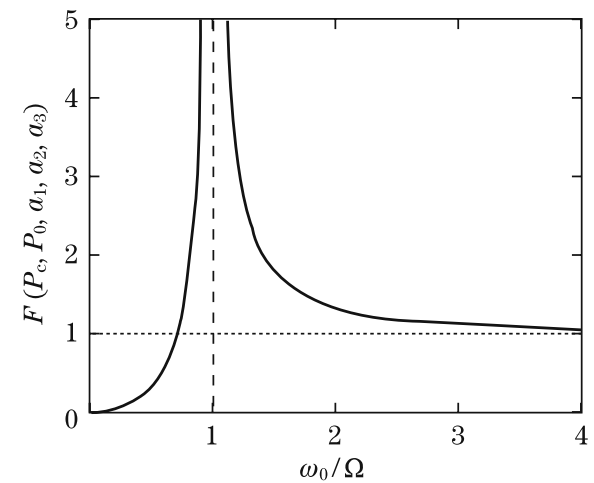

Fig. 3 Frequency response diagram

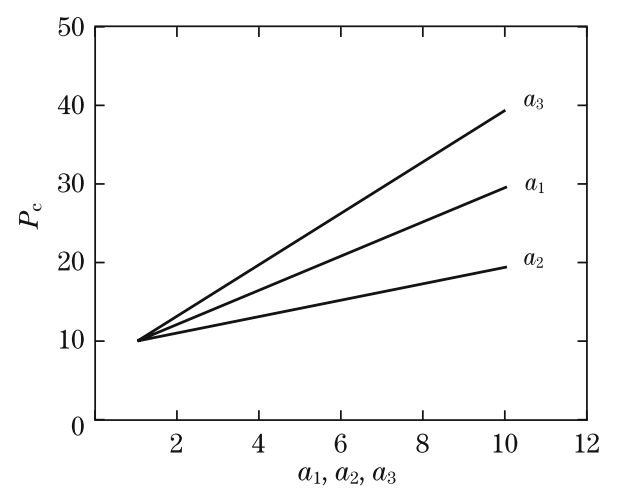

Fig. 4 Variation of $P_{\mathrm{c}}$ with $a_{1}, a_{2}$ and $a_{3}$ for frequency ratio $\omega_{0} / \Omega=0.6$ and static load $P_{0}=1$

It can be observed from Fig. 5 that the static load $P_{0}$ and $a_{3}$ have the same influence on $P_{\mathrm{c}}$. Furthermore, as expected, the maximum value $P_{\mathrm{c}}$ is reached at the maximum values of $P_{0}$ and $a_{3}$. It is obvious that in Fig. $6, P_{\mathrm{c}}$ decreases as the frequency ratio increases, and $a_{3}$ increases with the increase of $P_{\mathrm{c}}$. It can also be observed that the maximum $P_{\mathrm{c}}$ is reached at the lowest frequency ratio and at the maximum $a_{3}$ value. 
To clarify the effects of system parameters on the stability, the stability-instability charts (see Fig. 7) related with the static parameter $d_{1}$ and dynamic parameters $d_{2 \mathrm{c}}, d_{2 \mathrm{~s}}$, and $d_{2 \mathrm{~d}}$ when $k=0.5$ are obtained by using the numerical solution to Eq. (12). Shaded regions appearing in the figures represent the stable solution to Eq. (12). In Fig. 7(c), the stability region is considerably different from Figs. 7(a) and 7(b) because of the most effective parameter $a_{3}$. In such a case, the effect of $a_{3}$ increases, and the stable region is enlarged. It is also noted that the stable regions are moderately different from those given in [5] since the driving force is assumed as the linear combination of square of the elliptic functions $\operatorname{cn}\left(\tau, k^{2}\right), \operatorname{sn}\left(\tau, k^{2}\right)$, and $\operatorname{dn}\left(\tau, k^{2}\right)$ in the present study.

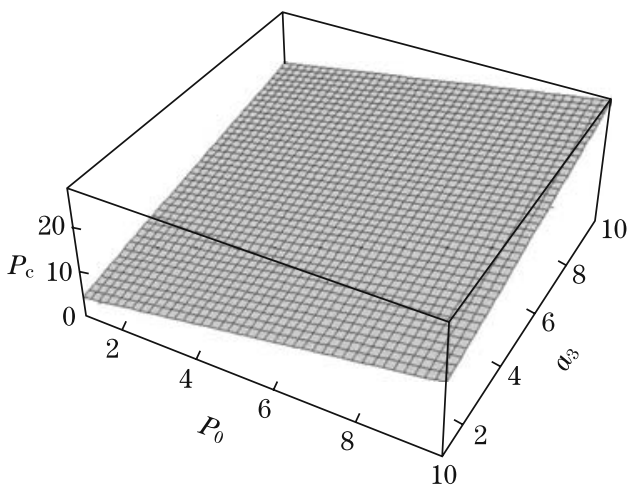

Fig. 5 Effects of $P_{0}$ and $a_{3}$ on $P_{\mathrm{c}}$ with driving forces $a_{1}=1, a_{2}=1$, and $\omega_{0} / \Omega$ $=1$

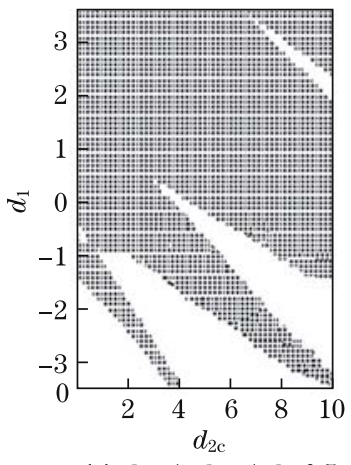

(a) $d_{2 \mathrm{~s}}=1, d_{2 \mathrm{~d}}=1, k=0.5$

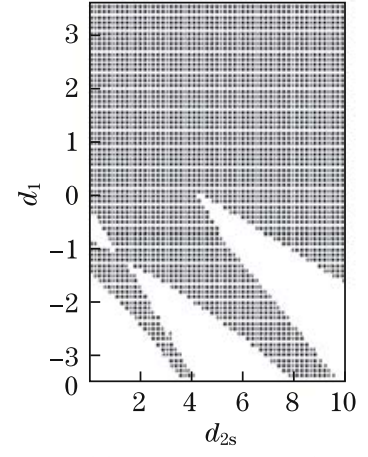

(b) $d_{2 \mathrm{c}}=1, d_{2 \mathrm{~d}}=1, k=0.5$

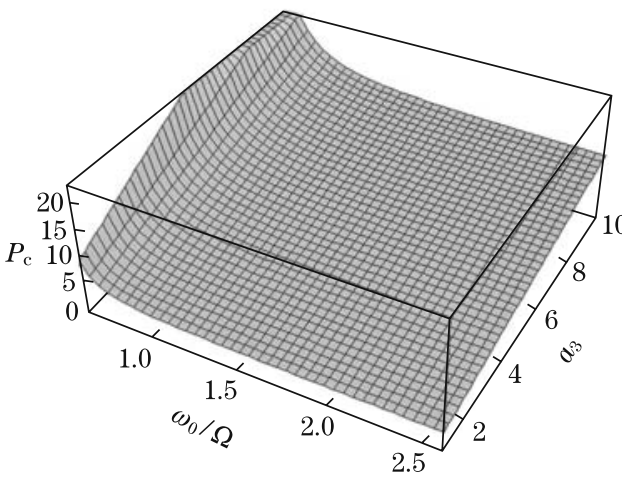

Fig. 6 Effects of frequency ratio $\omega_{0} / \Omega$ and driving force $a_{3}$ on $P_{\mathrm{c}}$ with $a_{1}=1$, $a_{2}=1$

Fig. 7 Stability-instability charts for Eq. (12)

\section{Conclusions}

In this paper, a closed form solution to the dynamic stability problem of a beam-column system loaded by an axial force of an elliptic type is presented. The frequency response characteristics of the system are obtained for different cases. The effects of the static load, driving forces, and frequency ratio on the Euler load are discussed. Stability-instability charts are also investigated for the Lamé equation (Eq. (12)). It can be concluded that the particular solution in [5] can only be used in the case of $a_{2}=a_{3}=0$ corresponding to the equation

$$
\frac{d^{2} u_{1}}{d \tau^{2}}+u_{1}(\tau)\left(d_{1}+d_{2 \mathrm{c}} \mathrm{cn}^{2}\left(\tau, k^{2}\right)\right)=0
$$


However, the particular solution given in this study is an appropriate solution to both the following two equations:

$$
\begin{aligned}
& \frac{d^{2} u_{1}}{d \tau^{2}}+u_{1}(\tau)\left(d_{1}+d_{2 \mathrm{c}} \mathrm{cn}^{2}\left(\tau, k^{2}\right)+d_{2 \mathrm{~s}} \operatorname{sn}^{2}\left(\tau, k^{2}\right)+d_{2 \mathrm{~d}} \mathrm{dn}^{2}\left(\tau, k^{2}\right)\right)=0 \\
& \frac{d^{2} u_{1}}{d \tau^{2}}+u_{1}(\tau)\left(d_{1}+d_{2 \mathrm{c}} \mathrm{cn}^{2}\left(\tau, k^{2}\right)\right)=0
\end{aligned}
$$

It is also found that $a_{3}$ is the most effective loading parameter for the presented problem.

\section{References}

[1] Bazant, Z. P. and Cedolin, L. Stability of Structures: Elastic, Inelastic, Fracture, and Damage Theories, Dover Publications, Inc., New York (2003)

[2] Aristizabal-Ochoa, J. D. Satic and dynamic stability of uniform shear beam-columns under generalized boundary conditions. Journal of Sound and Vibration 307(1-2), 69-88 (2007)

[3] Pavlovic, R., Kozic, P., Rajkovic, P., and Pavlovic, I. Dynamic stability of a thin-walled beam subjected to axial loads and end moments. Journal of Sound and Vibration 301(3-5), 690-700 (2007)

[4] Sampaio, J. H. B. and Hundhausen, J. R. A mathematical model and analytical solution for buckling of inclined beam-columns. Applied Mathematical Modelling 22(6), 405-421 (1998)

[5] Zuniga, A. E. Analysis of a beam-column system under varying axial forces of elliptic type: the exact solution of Lame's equation. International Journal of Solids and Structures 41(8), 2155-2163 (2004)

[6] Abramowitz, M. and Stegun, I. A. Handbook of Mathematical Functions with Formulas, Graphs, and Mathematical Tables, Dover Publications, Inc., New York (1965)

[7] Polyanin, A. D. and Zaitsev, V. F. Handbook of Exact Solutions for Ordinary Differential Equations, CRC Press, Boca Raton (1995)

[8] Lawden, D. F. Elliptic Functions and Applications, Springer-Verlag, New York (1989) 